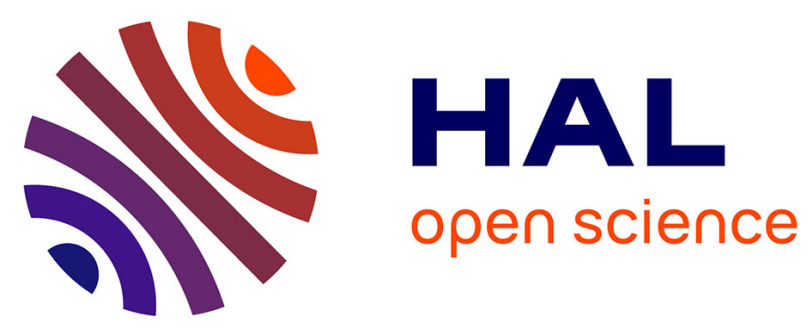

\title{
Spatial Data Fusion for Qualitative Estimation of Fuzzy Request Zones: Application on Precision Viticulture
} Jean-Noël Paoli, Olivier Strauss, Bruno Tisseyre, Jean-Michel Roger, Serge Guillaume

\section{- To cite this version:}

Jean-Noël Paoli, Olivier Strauss, Bruno Tisseyre, Jean-Michel Roger, Serge Guillaume. Spatial Data Fusion for Qualitative Estimation of Fuzzy Request Zones: Application on Precision Viticulture. Fuzzy Sets and Systems, 2007, 158 (5), pp.535-554. 10.1016/j.fss.2006.10.019 . lirmm-00127934

\section{HAL Id: lirmm-00127934 \\ https://hal-lirmm.ccsd.cnrs.fr/lirmm-00127934}

Submitted on 30 Apr 2007

HAL is a multi-disciplinary open access archive for the deposit and dissemination of scientific research documents, whether they are published or not. The documents may come from teaching and research institutions in France or abroad, or from public or private research centers.
L'archive ouverte pluridisciplinaire HAL, est destinée au dépôt et à la diffusion de documents scientifiques de niveau recherche, publiés ou non, émanant des établissements d'enseignement et de recherche français ou étrangers, des laboratoires publics ou privés. 


\title{
Spatial data fusion for qualitative estimation of fuzzy request zones: Application on precision viticulture
}

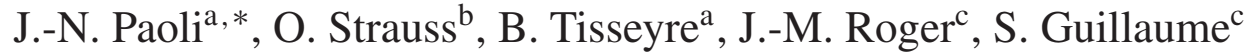 \\ ${ }^{a}$ Ecole Nationale Supérieure Agronomique, UMR ITAP, 2 place Viala, 34070 Montpellier cedex 1, France \\ ${ }^{\mathrm{b}}$ Laboratoire d'informatique, de Robotique, et de Microélectronique, 161 Rue Ada, 34392 Montpellier Cedex 5, France \\ ${ }^{\mathrm{c}}$ Cemagref, UMR ITAP, 361 rue Breton, 34033 Montpellier cedex 1, France
}

Received 22 February 2005; received in revised form 16 October 2006; accepted 17 October 2006

Available online 17 November 2006

\begin{abstract}
This article deals with a method used to describe and manage spatial knowledge. Each spatial datum is considered as an information element, whose location and value are independently described. The method proposed uses the information elements to infer the value of any request zone. Data can be either qualitative or quantitative. Our approach is based on a fuzzy granulation of available items of information. The fusion of information elements is performed by an aggregation operator based on the Choquet integral, which analyzes the spatial relevancy of the information elements on the request zone. We also describe an application of the method to a precision viticulture data set.

(c) 2006 Elsevier B.V. All rights reserved.
\end{abstract}

Keywords: Spatial information; Possibility theory; Choquet integral; Precision viticulture

\section{Introduction}

During the last decade, spatial knowledge management has become increasingly popular in many application fields: geographics, soil sciences, precision agriculture, etc. New technologies, such as remote sensing [25,26], or sensors associated with GPS (global positioning system) receivers [3], generate a large amount of spatial data. These data can be used to generate mono-variable maps (such as soil properties maps [14]) or to feed multi-variables decision support systems (such as systems used to choose fertilization strategies in agriculture [22]).

In precision viticulture, professionals (such as wine growers) typically want to aggregate different data in order to understand variability $[41,10,45,46]$ and for site-specific management [47]. Fig. 1 presents an example of a spatial data set. Firstly, grape yield (Fig. 1a) is measured by a monitoring system mounted on a grape harvester. Secondly, trunk diameters measurements are carried out manually (Fig. 1b). Thirdly, soil depths are estimated by a wine-grower (Fig. 1c). The associated locations are computed using GPS receivers (for grape yield and trunk diameter) or approximately defined from expert knowledge (for soil depth). The wine grower strives to aggregate these data to identify water availability problems and to plan site-specific irrigation.

\footnotetext{
* Corresponding author. Tel.: +33680415154.

E-mail address: paoli@ensam.inra.fr (J.-N. Paoli).
} 
a

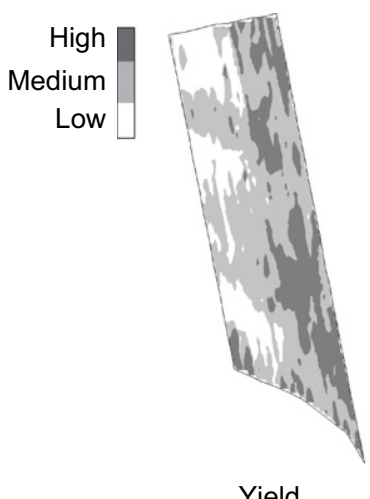

(2400 points/ha) b

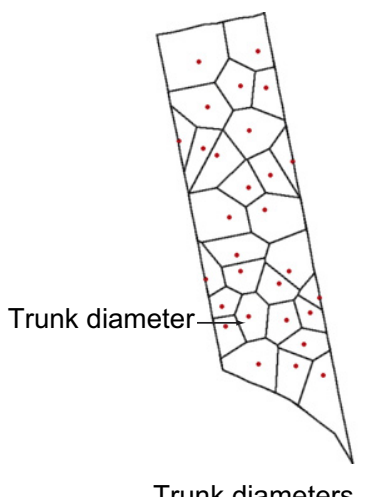

(30 points/ha)
C

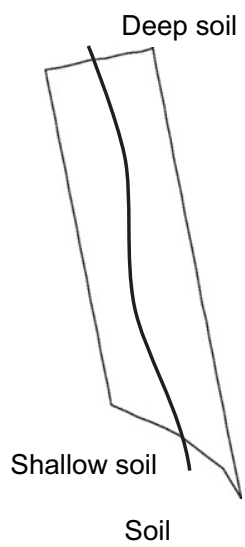

(2 expert zones)

Fig. 1. Yield map of a vineyard and complementary measurements: (a) yield (2400 points/ha); (b) trunk diameters (30 points/ha); (c) soil (two expert zones).

This example illustrates how spatial data may be naturally heterogeneous. Some values are qualitative (described in linguistic terms), such as soil depth, while some others are quantitative (described with numerical data), such as grape yield or trunk diameter. It shows that the amount of spatial data and its distribution are variable. Indeed, Fig. 1 shows:

- some yield zones computed from 2400 grape yield measurements,

- 30 points which correspond to the locations of the trunk diameter measurements,

- two soil depth zones (bounded by a single line).

In addition, there are other differences between the data. The precision and the expected confidence are also heterogeneous. Yield values and locations are imprecise because of the sensors used to determine them. Each value can be associated with a bounded error (1-2\% of the registered value for the yield sensor [8], 1-3 $\mathrm{m}$ for the GPS receiver [23]). Trunk diameter measurements are intrinsically imprecise and uncertain, because of the within-stock variability. The expert partition of the vineyard is quite rough. Each zone of the partition is associated with linguistic terms (such as "the soil is shallow in the higher part of the vineyard"). In most cases, spatial continuity of the measured variables is not compatible with their representation given by precise boundaries [11,43,17]. For instance, there is usually a continuous transition zone between a shallow soil zone and a deep soil zone.

To plan site-specific irrigation, the wine grower is able to divide the vineyard into zones which can be irrigated independently from the others (for more details about management zones, see Doerge [16] and Kvien and Pocknee [31]). The shape of these "irrigation zones" is arbitrarily delineated based on technical criteria. This delineation is carried out without taking into account available data, and is not associated with any hypothesis on the studied variables (such as homogeneity). This delineation is necessarily imprecise as there is a continuous transition zone between an irrigated zone and a non-irrigated zone.

For each zone, the decision to irrigate or not depends on a qualitative estimate of the studied variables, and on the use of logical rules. Therefore, we need a method that can be used to generate qualitative estimates of studied variables on an arbitrarily defined partition of a field.

This paper defines a new approach to spatial estimation. We propose a semantic based aggregation method (SA method). It aims at providing a classification of geographic zones by aggregating quantitative and qualitative pieces of information and accounting for both precision and confidence (if known). It is based on the granulation of the available pieces of information on partitions that are semantically related to the sought-after classification. It integrates fuzzy sets, possibility degrees, and fuzzy integrals.

Section 2 presents a review of related works. It deals with methods used to model spatial information with fuzzy sets, and to estimate spatial data. The semantic based aggregation method is defined in Section 3. An application of this method on precision viticulture data is presented in Section 4. We characterize within field variations of vineyards. 
We used the method in the way that it would be used by a technician or a wine-grower, to determine the response of a parameter (such as the yield), considering the other parameters (the vigor, the elevation, etc.). This example illustrates the ability of the proposed method to consider heterogeneous data. The main conclusions are stated in Section 5.

\section{Related work}

\subsection{Fuzzy spatial data}

In the literature, spatio-temporal data in GIS (geographical information systems) are defined using three components: location, measurement (attribute), and time. Each component can be considered imprecise and uncertain [17]:

- The fuzzy set theory [49] has been extensively used to describe attribute imprecision and uncertainty $[52-54,35,32,12,47,2]$.

- The description of spatial vagueness is based on the definition of spatial objects isolated from the rest of the space by imprecise boundaries. These objects can be described using probabilistic or fuzzy models [11]. Fuzzy spatial data types (fuzzy points, fuzzy lines, and fuzzy polygons) are defined using specific fuzzy subsets of the geographical space $[43,38]$.

- Fuzzy sets can also be used to define the time component. Dragićević and Marceau [18] associate fuzzy subsets of the time referential with snapshot layers in a raster GIS database.

Different types of geographic data can be defined using a combination of crisp and fuzzy components [17]. This work only considers spatial data, defined by crisp or fuzzy attributes and crisp or fuzzy locations. The time component is never taken into account.

\subsection{Fuzzy spatial estimates}

Geographic phenomena are usually described by discrete spatial data sets. The unknown value of a variable at a given location has to be estimated using available data. The method used to provide these estimates depends on available data. Linear interpolations and splines are commonly applied to crisp spatial data. Fuzzy linear interpolations [21] and fuzzy splines [1] can be used to interpolate spatial data defined using fuzzy attributes [33].

Geostatistics and kriging methods are extensively used to aggregate crisp spatial data $[36,28,29,9]$. These methods provide linear interpolations of the known values. However, they are based on a statistical study of spatial variations. A function called variogram is modeled to describe these variations. It is used to weigh the known values and to associate variances (called "kriging variances") with the estimates. If one considers that the variogram model is valid, these methods provide the best possible linear estimators. However, to compute a variogram, at least a 100 points are needed with the method of moments, and a minimum of 50 points are needed with maximum likelihood approaches [30]. Therefore, many spatial data sets cannot be considered because they only contain a small information set (such as 30 trunk diameter measurements, in the example presented in Fig. 1).

Fuzzy kriging [5,15] can be applied to spatial data, which are defined with a fuzzy attribute. Diamond worked on spatially distributed fuzzy data modeled by a random function taking values that are triangular fuzzy numbers (a similar approach can be applied to trapezoidal fuzzy numbers). The random function is described using modal, upper and lower variogram functions. These three functions are aggregated to compute crisp kriging variances. Fuzzy kriging was used to model glacial thickness to a regional scale [42].

Bandemer and Gebhardt [4] combined fuzzy kriging and bayesian linear kriging. The field is defined by adding a fuzzy general trend to a fuzzy stochastic model (universal fuzzy kriging). The general trend is parameterized with a fuzzy random variable. The bayesian approach is used to define this fuzzy random variable.

Fuzzy variograms have been defined [6] to account for the imprecision and uncertainty of the variogram model. Let $\gamma$ be an analytical variogram model defined by a parameter vector $p$. Each vector $p$ is associated with a membership degree in the set of all the possible parameter vectors $\mathcal{P}$. This membership degree shows how acceptable it is. In this case, the random function is defined using a fuzzy set of all the possible variograms. It is used to compute fuzzy kriged estimates and fuzzy kriging variances. 


\subsection{Semantic based aggregation method}

All these usual methods are not relevant when only a small set of data are available, or when the measured values are imprecise or uncertain. Moreover, they do not address qualitative pieces of information and do not have any ability to account for expert knowledge.

In the next section, we propose a semantic based aggregation method (SA method) which does not consider numerical values but rather qualitative and quantitative pieces of information granulated on partitions associated with semantic labels. Our approach also differs from usual approaches as it takes into account the quantity of available information. Indeed, it is not based on the underlying hypothesis that the available data set is big enough to determine the entire field. Each datum describes a subarea of the field. Each subarea is defined independently from the others. Some parts of the field can be described by several data, whereas some others cannot be described by any datum. In our approach, the quantity of available information on the subarea to be estimated is used to improve the relevancy of the estimate.

\section{The estimation process}

\subsection{Definitions}

This subsection is dedicated to the definition of spatial information elements and presents the objective of the estimation process. A spatial information element is defined using a location component (a fuzzy subset of the geographical space) and a measurement component (possibility and necessity degrees associated with qualitative labels). Regions of particular interest are called request zones. They can be regarded as information elements whose value component is unknown.

\subsubsection{Referential}

A spatial information element is defined using two components: location and measurement. These two components are considered to be independent from one another. The location component is defined on a referential $G$ (for instance $\left.G=\left(\mathbb{R}^{+}\right)^{2}\right)$. The measurement component is associated with a frame of reference $M$ (for example $M=[0,20]$ ).

As mentioned in the previous sections, we aim at aggregating qualitative and quantitative pieces of information. We propose to convert all quantitative data into qualitative pieces of information. This conversion is based on the association of linguistic terms (such as low, medium, or high) with fuzzy subsets of the referential $M$.

Let $\mathcal{U}(M)$ be a set of labels related to a fuzzy partition of $M$ :

$$
\mathcal{U}(M)=\left\{\left(A_{i}\right), i \in[1, \ldots, p]\right\} .
$$

$\mathcal{U}(M)$ is the set of all the labels the measurement component can take (for instance, $A_{1}=$ low, $A_{2}=$ medium, $A_{3}=$ high).

\subsubsection{The spatial information element}

The location component is a subset of the geographical space. It defines the location from where the information has been collected and its spatial extend (i.e. the neighboring area within which the measurement remains valid). Experts may have a rough idea of the spatial variability of a variable, and are therefore able to enlarge the spatial extend of each datum.

The measurement component is provided by a sensor or expert knowledge. It is defined by possibility and necessity degrees $[50,20]$ associated with the qualitative labels in $\mathcal{U}(M)$. This approach is useful when attempting to manage qualitative and quantitative data within the same framework. It is based on a granulation approach, which is a simple concept, based on human cognition, involving decomposition of the whole into parts [51].

The association of a possibility degree with a necessity degree can be seen as a bipolar confidence measure [7], which accounts for the fuzzy definition of the labels in $\mathcal{U}(M)$ and for both imprecision and uncertainty of the measurement. 
A spatial information element $I$ is a discrete entity that restricts $G \times \mathcal{U}(M)$. This restriction is defined by

$$
I=\left(\mathrm{P}(I), \Pi\left(A_{i} ; I\right), \mathrm{N}\left(A_{i} ; I\right)\right), \quad i \in[1, \ldots, p] .
$$

- $\mathrm{P}(I)$ is a fuzzy subset of $G$,

- $\Pi\left(A_{i} ; I\right)$ and $\mathrm{N}\left(A_{i} ; I\right)$ are, respectively, the possibility and necessity degree of $A_{i}$ restricted to $I$. They are defined by

$$
\begin{aligned}
& \Pi\left(A_{i} ; I\right)=\sup _{m \in M} \min \left(\mu_{\mathrm{V}(I)}(m), \mu_{A_{i}}(m)\right), \\
& \mathrm{N}\left(A_{i} ; I\right)=\inf _{m \in M} \max \left(\mu_{\mathrm{V}(I)}(m), 1-\mu_{A_{i}}(m)\right)
\end{aligned}
$$

with:

$\circ \mathrm{V}(I)$ a fuzzy subset of $M$,

○ $\mu_{\mathrm{V}(I)}: M \rightarrow[0,1]$ the membership function of $\mathrm{V}(I)$,

○ $\mu_{A_{i}}: M \rightarrow[0,1]$ the membership function of $A_{i}$.

\subsubsection{Request zone}

A request zone is a region of $G$ for which analysis has to be carried out, and also a region where a site-specific management has to be implemented. From a theoretical point of view, a request zone can be considered as a spatial information element whose measurement component is unknown.

Let $\mathrm{P}(R)$ be a fuzzy subset of $G$ modeling a request zone. $R$ is the spatial information element, whose location is described by the fuzzy subset $\mathrm{P}(R)$.

$$
R=\left(\mathrm{P}(R), \Pi\left(A_{i} ; R\right), \mathrm{N}\left(A_{i} ; R\right)\right), \quad i \in[1, \ldots, p] .
$$

\subsection{Estimates on request zones of possibility and necessity degrees}

The fusion of the available spatial data aims at providing an estimate of the value component of $R$, i.e. at computing upper and lower bounds of confidence degrees from the possibility and necessity degrees provided by the available data. This subsection proposes a method to provide robust estimates of these bounds. This method is based on a $\beta$-percentile like operator. Its utility resides in the fact that it accounts for both imprecision and uncertainty.

\subsubsection{The preliminary concept: fuzzy measures and fuzzy integrals}

A fuzzy measure is a monotonic non-additive function. An aggregation of values with respect to a fuzzy measure can be computed with a fuzzy integral $[44,34,24]$. The Choquet integral is one of the fuzzy integrals at our disposal. It was proposed by Murofushi and Sugeno [39] and is based on the capacity concept introduced by Choquet [13].

Let $S$ be the set of elements to be aggregated. Let $F$ be a fuzzy measure defined by

$$
\begin{aligned}
& 2^{S} \rightarrow[0,1], \\
& T \rightarrow F(T), \\
& F(\phi)=0, \\
& T_{2} \subseteq T_{1} \Leftrightarrow F\left(T_{2}\right) \leqslant F\left(T_{1}\right) .
\end{aligned}
$$

The aggregation data by a Choquet integral, with respect to a fuzzy measure $F$, is given by

$$
\begin{aligned}
& C_{F}(X)=\sum_{k=1}^{n} X_{(k)}\left[F\left(T_{(k)}\right)-F\left(T_{(k+1)}\right)\right], \\
& T_{(k)}=\left\{X_{(k)}, \ldots, X_{(n)}\right\}, \quad k \in[1, n], \\
& T_{(n+1)}=\phi .
\end{aligned}
$$


$\left\{X_{(1)}, \ldots, X_{(n)}\right\}$ is a permutation of $\left\{X_{1}, \ldots, X_{n}\right\}$ such that

$$
X_{(1)} \leqslant X_{(2)} \leqslant \cdots \leqslant X_{(n)} .
$$

Fuzzy measures and fuzzy integrals are commonly used to define aggregation operators, accounting for interactions between the information elements to be aggregated. They can also be used to generalize to fuzzy sets some usual aggregation operators defined for crisp sets. The values computed for each $\alpha$-cut can be aggregated using a Choquet integral in relation to the fuzzy measure $\alpha[19]$.

\subsubsection{Set of the relevant information sources}

Let $S$ be a set of information elements, called information sources.

$$
S=\left\{I_{k}, k \in[1, \ldots, n]\right\} .
$$

The spatial compatibility between the information sources $\left\{I_{1}, I_{2}, \ldots, I_{n}\right\}$ and the request zone $R$ depends on their locations. The spatial compatibility between $R$ and a single information source $I_{k}$ is described by a possibility of interaction $\Pi\left(\mathrm{P}(R) ; \mathrm{P}\left(I_{k}\right)\right)$.

Let $S^{\prime}$ be the set of relevant information sources. $S^{\prime}$ is a fuzzy subset of $S$ defined by its membership function $\mu_{S^{\prime}}$ :

$$
\begin{aligned}
& \mu_{S^{\prime}}: S \rightarrow[0,1], \\
& \mu_{S^{\prime}}\left(I_{k}\right)=\Pi\left(\mathrm{P}(R) ; \mathrm{P}\left(I_{k}\right)\right) .
\end{aligned}
$$

\subsubsection{Principle of the estimate}

We wish to define upper and lower bounds of the confidence degree of $A_{i}$ on the request zone, by aggregating possibility and necessity degrees $\left(\Pi\left(A_{i} ; I_{k}\right)\right.$ and $\left.\mathrm{N}\left(A_{i} ; I_{k}\right)\right)$, and by accounting for the spatial compatibility between the information sources and the request zone. These upper and lower bounds will be denoted $\widehat{\Pi}\left(A_{i} ; R\right)$ and $\widehat{\mathrm{N}}\left(A_{i} ; R\right)$.

Because no hypothesis can be formulated on the underlying distribution of the data to be aggregated, this aggregation operator should be as distribution free as possible. This leads us to robust non-parametric aggregating operators (i.e. insensitive to small deviations of assumptions on the data [27]).

A first approach could be to consider the direct computation of the possibility and necessity degrees $\left(\Pi\left(A_{i} ; I_{k}\right)\right.$ and $\left.\mathrm{N}\left(A_{i} ; I_{k}\right)\right)$ using a Choquet integral [19]. It leads to the following definition:

$$
\begin{aligned}
& \widehat{\Pi}\left(A_{i} ; R\right)=\Pi\left(A_{i} ; R\right)=\int_{0}^{1} \sup _{k}\left\{\Pi\left(A_{i} ; I_{k}\right) \mid \Pi\left(\mathrm{P}(R) ; \mathrm{P}\left(I_{k}\right)\right) \geqslant \alpha\right\} \mathrm{d} \alpha, \\
& \widehat{\mathrm{N}}\left(A_{i} ; R\right)=\mathrm{N}\left(A_{i} ; R\right)=\int_{0}^{1} \inf _{k}\left\{\mathrm{~N}\left(A_{i} ; I_{k}\right) \mid \Pi\left(\mathrm{P}(R) ; \mathrm{P}\left(I_{k}\right)\right) \geqslant \alpha\right\} \mathrm{d} \alpha .
\end{aligned}
$$

Note that $\Pi\left(A_{i} ; R\right)$ and $\mathrm{N}\left(A_{i} ; R\right)$ are possibility and necessity degrees. However, this method is very sensitive to outliers as a single outlier observation makes it generate upper and lower bounds that are not very informative.

Let us take a simple data set containing nine relevant values and one outlier observation. Let $S$ be a set of yield values collected on an experimental vineyard. For nine information elements, the yield is high $(\Pi(h i g h)=1$ and $\mathrm{N}($ high $)=1)$. For one information element (collected on a dead stock of vine), the yield is low $(\Pi($ low $)=1$ and $\mathrm{N}($ low $)=1)$. We would like the aggregating operator to provide: $\Pi($ high $)=1$ and $\mathrm{N}($ high $)=1$.

The direct estimation of possibility and necessity degrees leads to: $\Pi($ high $)=1$ and $\mathrm{N}($ high $)=0(\Pi($ low $)=1$ and $\mathrm{N}($ low $)=0)$. This result is relevant but less informative than hoped.

A second aggregative approach is to consider the usual weighted mean. $\widehat{\Pi}\left(A_{i} ; R\right)$ and $\widehat{\mathrm{N}}\left(A_{i} ; R\right)$ are upper and lower bounds of confidence measures, as $\widehat{\Pi}\left(A_{i} ; R\right) \geqslant \widehat{\mathrm{N}}\left(A_{i} ; R\right)$. However, the fact that $\widehat{\Pi}\left(A_{i} ; R\right)$ and $\widehat{\mathrm{N}}\left(A_{i} ; R\right)$ are no longer possibility and necessity degrees must be noted.

$$
\widehat{\Pi}\left(A_{i} ; R\right)=\frac{\sum_{k} \Pi\left(A_{i} ; I_{k}\right) \Pi\left(\mathrm{P}(R) ; \mathrm{P}\left(I_{k}\right)\right)}{\sum_{k} \Pi\left(\mathrm{P}(R) ; \mathrm{P}\left(I_{k}\right)\right)},
$$




$$
\widehat{\mathrm{N}}\left(A_{i} ; R\right)=\frac{\sum_{k} \mathrm{~N}\left(A_{i} ; I_{k}\right) \Pi\left(\mathrm{P}(R) ; \mathrm{P}\left(I_{k}\right)\right)}{\sum_{k} \Pi\left(\mathrm{P}(R) ; \mathrm{P}\left(I_{k}\right)\right)} .
$$

The results provided by this method are more specific than those provided by the first one. However, weighted sum based aggregation processes are known to be highly sensitive to outliers [27]. Indeed, spurious outlier observations lead to inconsistent estimates. With the example previously defined, this operator provides: $\Pi(h i g h)=0.9$ and $\mathrm{N}(h i g h)=0.1$ $(\Pi($ low $)=0.1$ and $\mathrm{N}($ low $)=0.1)$. This result is not relevant as a low yield hypothesis could be taken into account for the management of the field.

Therefore we propose a $\beta$-percentile based aggregation operator, which is a robust $L$-estimator [27]. Like for the weighted mean approach, upper and lower bounds of confidence degrees computed with this kind of operators are not possibility and necessity degrees. However, $\beta$-percentile produces more specific results than the usual possibilistic aggregation and the presence of outliers does not lead to inconsistent estimates.

Nevertheless, this operator is usually used to aggregate crisp sets of values. In Section 3.2.4, we will propose a definition of this operator based on the use of a Choquet integral. In Section 3.2.5, we will propose a generalization of this $\beta$-percentiles to fuzzy sets of values.

\subsubsection{Definition of the $\beta$-percentile}

Let $A$ be the label to be associated with upper and lower confidence degrees. For simplicity sake, we will denote $\Pi_{k}=\Pi\left(A_{i}, I_{k}\right)$.

Let $S^{\prime}=\left\{\Pi_{1}, \ldots, \Pi_{n}\right\}$ be a set of values to be aggregated. $S^{\prime}$ is a crisp subset of $S$. $L_{\beta}(\Pi)$ is a $\beta$-percentile of $S^{\prime}$. It is defined by

$$
\begin{aligned}
& L_{\beta}(\Pi)=\sum_{k} a_{k}(\beta) \Pi_{(k)}, \\
& a_{k}>0 \Leftrightarrow k \in\left[n\left(\beta-\frac{\delta}{2}\right), n\left(\beta+\frac{\delta}{2}\right)\right], \\
& a_{k}=0 \Leftrightarrow k \notin\left[n\left(\beta-\frac{\delta}{2}\right), n\left(\beta+\frac{\delta}{2}\right)\right] .
\end{aligned}
$$

$\left\{\Pi_{(1)}, \ldots, \Pi_{(n)}\right\}$ is a permutation of $\left\{\Pi_{1}, \ldots, \Pi_{n}\right\}$ such that

$$
\Pi_{(1)} \leqslant \Pi_{(2)} \leqslant \cdots \leqslant \Pi_{(n)} .
$$

The value of $a_{k}$ only depends on the rank $k$ of $\Pi_{(k)}$ in the ordered set of values $\left\{\Pi_{(1)}, \ldots, \Pi_{(n)}\right\}$. $\beta$ defines the level of percentile (i.e. the rank of the aggregated value in $\left.\left\{\Pi_{(1)}, \ldots, \Pi_{(n)}\right\}\right)$ and $\delta$ controls the amount of filtering.

Murofushi and Sugeno [40] have shown that $L$-statistic like operators can be computed using Choquet integrals, with respect to fuzzy measures $F$ based on the ranks of the values to be aggregated.

$$
\begin{aligned}
& L_{\beta}(\Pi)=\sum_{k=1}^{n} \Pi_{(k)}\left[F\left(T_{(k)}\right)-F\left(T_{(k+1)}\right)\right], \\
& T_{(k)}=\left\{\Pi_{(k)}, \ldots, \Pi_{(n)}\right\} .
\end{aligned}
$$

A $\beta$-percentile like operator can be defined using a fuzzy measure $F$ (shown in Fig. 2) such that

$$
\begin{aligned}
& \begin{cases}F\left(T_{(k)}\right)=0 & \text { when } \rho_{(k)} \leqslant(1-\beta)-\frac{\delta}{2}, \\
F\left(T_{(k)}\right)=\frac{\rho_{(k)}-\beta}{\delta}+\frac{1}{2} & \text { when }(1-\beta)-\frac{\delta}{2} \leqslant \rho_{(k)} \leqslant(1-\beta)+\frac{\delta}{2}, \\
F\left(T_{(k)}\right)=1 & \text { when } \rho_{(k)} \geqslant(1-\beta)+\frac{\delta}{2},\end{cases} \\
& \rho_{(k)}=\frac{r\left(\Pi_{(n)}\right)-r\left(\Pi_{(k-1)}\right)}{r\left(\Pi_{(n)}\right)},
\end{aligned}
$$

where $r\left(\Pi_{k}\right)$ and $\rho(k)$ are, respectively, the rank and the relative rank of the value $\Pi_{k}$ in the ordered set $\Pi_{(1)} \leqslant \Pi_{(2)} \leqslant \cdots$ $\leqslant \Pi_{(n)}$. 


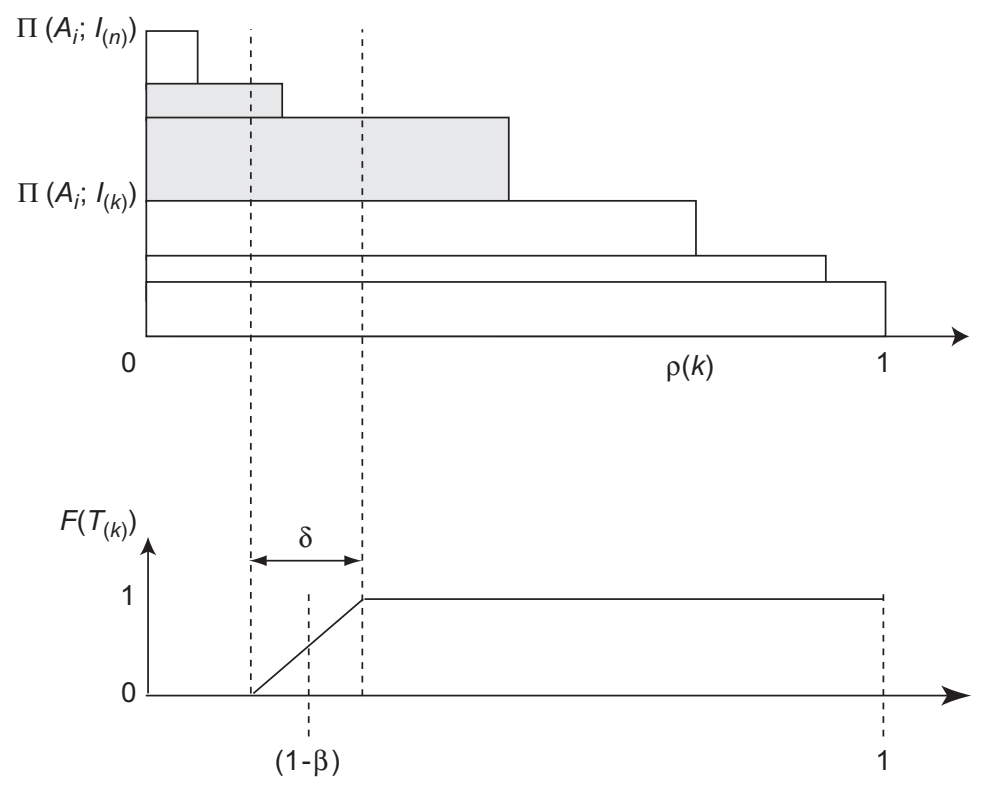

Fig. 2. Graphical representation of the fuzzy measure $F$.

The rank $r$ is defined as follows:

$$
\begin{aligned}
& \left\{\begin{array}{l}
\chi_{S^{\prime}}: S^{\prime} \rightarrow\{0,1\}, \\
\chi_{S^{\prime}}\left(\Pi_{k}\right)=0 \Leftrightarrow \Pi_{k} \notin S^{\prime}, \\
\chi_{S^{\prime}}\left(\Pi_{k}\right)=1 \Leftrightarrow \Pi_{k} \in S^{\prime}
\end{array}\right. \\
& r\left(\Pi_{k}\right)=\sum_{\Pi_{j} \leqslant \Pi_{k}} \chi_{S^{\prime}}\left(\Pi_{j}\right) .
\end{aligned}
$$

While $S^{\prime}$ is a crisp subset of $S$, formula (19) can be simplified as

$$
\begin{aligned}
& r\left(\Pi_{(k)}\right)=k, \\
& \rho_{(k)}=\frac{n-k+1}{n} .
\end{aligned}
$$

In the next section, $S^{\prime}$ will be considered as a fuzzy subset of $S$. This will mean generalizing the $\operatorname{rank} r\left(\Pi_{k}\right)$.

\subsubsection{Fuzzy definition of $r\left(\Pi_{k}\right)$}

Let $S^{\prime}$ be a fuzzy subset of $S$ and $S_{\alpha}^{\prime}$ be the $\alpha$-cut of $S^{\prime}$.

$$
S_{\alpha}^{\prime}=\left\{\Pi_{k} / \mu_{S_{\alpha}^{\prime}}\left(\Pi_{k}\right) \geqslant \alpha\right\} .
$$

The rank $r_{\alpha}$ of any value $\Pi_{k}$ in $S_{\alpha}^{\prime}$ is defined as previously by formula (21). The fuzzy rank $r$ of $\Pi_{k}$ in $S^{\prime}$ is computed by a Choquet integral:

$$
\begin{aligned}
r\left(\Pi_{k}\right) & =\int_{0}^{1} r_{\alpha}\left(\Pi_{k}\right) \mathrm{d} \alpha \\
& =\int_{0}^{1} \sum_{\Pi_{j} \leqslant \Pi_{k}} \chi_{S_{\alpha}^{\prime}}\left(\Pi_{j}\right) \mathrm{d} \alpha
\end{aligned}
$$




$$
\begin{aligned}
& =\sum_{\Pi_{j} \leqslant \Pi_{k}} \int_{0}^{1} \chi_{S_{\alpha}^{\prime}}\left(\Pi_{j}\right) \mathrm{d} \alpha \\
& =\sum_{\Pi_{j} \leqslant \Pi_{k}} \mu_{S^{\prime}}\left(\Pi_{j}\right) .
\end{aligned}
$$

The combination of formulas (19) and (25) leads to

$$
\rho_{(k)}=\frac{\sum_{j=k}^{n} \mu_{S^{\prime}}\left(\Pi_{j}\right)}{\sum_{j=1}^{n} \mu_{S^{\prime}}\left(\Pi_{j}\right)} .
$$

\subsubsection{Estimate of possibility and necessity degrees}

According to the previous section, the estimate of possibility degree is defined as follows:

$$
\widehat{\Pi}\left(A_{i} ; R\right)=\sum_{k=1}^{n} \Pi\left(A_{i} ; I_{(k)}\right)\left[F\left(T_{(k)}\right)-F\left(T_{(k+1)}\right)\right] .
$$

Let $\overline{A_{i}}$ be the complementary set of $A_{i}$. The estimate of the possibility degree of $\overline{A_{i}}$ is provided by

$$
\widehat{\Pi}\left(\bar{A}_{i} ; R\right)=\sum_{k=1}^{n} \Pi\left(\bar{A}_{i} ; I_{(k)}\right)\left[F\left(T_{(k)}\right)-F\left(T_{(k+1)}\right)\right] .
$$

Possibility degrees are to respect the following property:

$$
\widehat{\Pi}\left(A_{i} ; R\right)+\widehat{\Pi}\left(\bar{A}_{i} ; R\right) \geqslant 1 .
$$

Nevertheless, as pointed out earlier, the computation of the upper percentile does not guarantee that property (29) is respected. We propose to account for this problem by using post-normalization.

$$
\begin{aligned}
& \tilde{\Pi}\left(A_{i} ; R\right)=\max \left(\widehat{\Pi}\left(A_{i} ; R\right), 1-\widehat{\Pi}\left(\bar{A}_{i} ; R\right)\right), \\
& \tilde{\Pi}\left(\bar{A}_{i} ; R\right)=\max \left(\widehat{\Pi}\left(\bar{A}_{i} ; R\right), 1-\widehat{\Pi}\left(A_{i} ; R\right)\right) .
\end{aligned}
$$

The estimate of the necessity degree of $A_{i}$ is given by the relation

$$
\tilde{\mathrm{N}}\left(A_{i} ; R\right)=1-\tilde{\Pi}\left(\bar{A}_{i} ; R\right) .
$$

The use of this operator on the example defined in Section 3.2.3 can provide the expected result: $\Pi($ high $)=1$ and $\mathrm{N}($ high $)=1($ for instance with $\beta=0.75$ and $\delta=0.1)$.

\subsubsection{Choice of the parameters $\beta$ and $\delta$}

The choice of $\beta$ and $\delta$ is usually based on expert knowledge. We take $(1-\beta)$ to be the fraction of information provided by outliers. $\delta$ is related to the quantity of spatial information required for an accurate estimate of the measurement component on the request zone. However, its choice is not straightforward because it depends on the number of available information elements. Therefore, we propose to compute $\delta$ from a quantity of spatial information $\Delta$, which can be easily derived from expert knowledge.

$$
\Delta=\frac{\delta}{\sum_{j=1}^{n} \mu_{S^{\prime}}\left(\Pi_{j}\right)} .
$$

Obviously $\Delta \geqslant 1$ because the quantity of spatial information required to estimate a possibility degree has to be at least equivalent to the quantity provided by a single relevant information source.

The value of $\beta$ depends on the value of $\delta$. According to the definition of the fuzzy measure $F$ :

$$
\beta-\frac{\delta}{2} \geqslant 0
$$




\subsubsection{Definition of the estimate when the quantity of information is too low}

If $\Delta>\sum_{j=1}^{n} \mu_{S^{\prime}}\left(\Pi_{j}\right)$, i.e. if the quantity of information required to define a request zone is higher than the quantity of available information, there is no value of $\beta$ such as

$$
\frac{1}{2} \leqslant \beta \leqslant 1-\frac{\delta}{2}
$$

In this case, we propose to parameter the estimate with

$$
\beta=\frac{\delta}{2} \text {. }
$$

This parameter ensures

$$
\begin{aligned}
& \beta-\frac{\delta}{2}=0, \\
& \beta+\frac{\delta}{2} \geqslant 1 .
\end{aligned}
$$

This parameter does not ensure that the operator provides an upper estimate. Indeed, the estimated value should be bounded by the fuzzy measure:

$$
\widehat{\Pi}\left(A_{i} ; R\right) \leqslant F\left(S^{\prime}\right) .
$$

However, this problem can be addressed by using post-normalization (30) and (31). Indeed, these relations increase the estimated possibility degrees, and decrease the estimated necessity degrees. There are no qualitative labels which can be considered as certain. The lack of information is translated into uncertainty.

In this section, we proposed a semantic based aggregation method (SA method) to provide a possibilistic classification of request zones. This method considers qualitative and quantitative pieces of information granulated on partitions associated with semantic labels. These granulated pieces of information are defined by possibility and necessity degrees. The granulation is useful for managing qualitative and quantitative knowledge within the same framework. The use of possibility and necessity degrees allows to account for both imprecision and uncertainty.

The estimation process provides lower and upper bounds of confidence degrees, for any qualitative label, on any request zone. It is based on a robust $\beta$-percentile like operator. The method is original because it considers fuzzy sets of spatial information elements, resulting from the intersection of fuzzy location components with fuzzy request zones.

Note that in this approach, spatial interactions between information sources (which could be described by $\Pi\left(\mathrm{P}\left(I_{k}\right)\right.$; $\left.\mathrm{P}\left(I_{l}\right)\right)$ ) are not taken into account. Further work is needed to study their importance and possible utility for method improvement.

\section{Application to precision viticulture data}

In this section, we apply our approach to precision viticulture data sets. Firstly, we test the ability of our method to generate maps. Secondly, we wish to assess the potential of our approach to aggregate some layers of spatial information. The section is organized as follows:

The first subsection presents the experimental vineyard, lists the available data and describes how these information sources are modeled.

The second subsection is dedicated to the comparison of the SBA method with usual methods (kriging and fuzzy kriging). This comparison is based on the computation of grape yield maps.

The third subsection highlights the ability of our method to account for expert knowledge. It presents a method used to validate an expert partition of the yield map into two management zones. A sensitivity analysis is carried out to characterize the influence of the parameters on the aggregation process. The results of this analysis are presented for one request zone. 
a

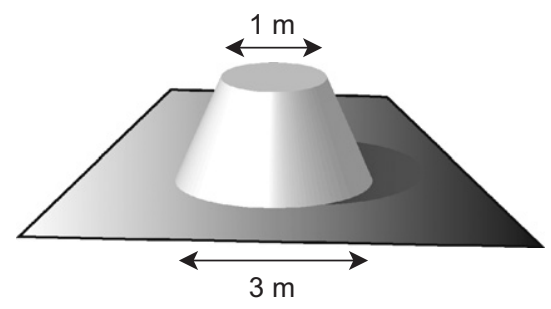

Spatial inaccuracy resulting from the dGPS b

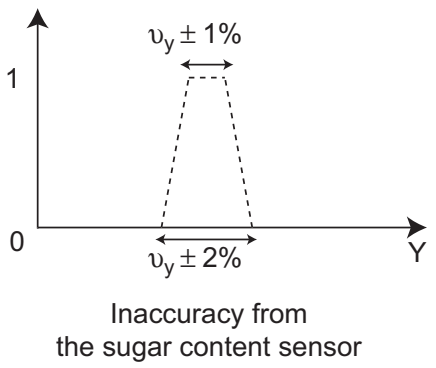

Fig. 3. Description of an information source: (a) spatial inaccuracy resulting from the DGPS; (b) inaccuracy from the sugar content sensor.

The fourth subsection presents a qualitative estimate of each variable on each expert zone using heterogeneous data sets (sugar content, elevation, vigor, soil resistivity and soil zones).

\subsection{Materials}

\subsubsection{Experimental data sets}

A Merlot variety is grown on our experimental vineyard, located in Navarra (winery Julian Chivite, Spain). The field area is 1.5 ha. Grape yield and sugar content were measured and mapped in September 2003 using an on-line sensor mounted on a grape-harvester (Pellenc S.A.) located by a DGPS (differential global positioning system). The resolution of the data is 2400 points/ha.

Complementary measurements were done in March 2004. Three main parameters were assessed at the within field level in order to explain within field variability. Elevation (157 measurements), trunks diameters (45 measurements), and soil resistivity (45 measurements) have been recorded. Two expert zones were also delimited within the field. The first one corresponds to a deep soil while the second one is characterized by a shallow soil.

\subsubsection{Information sources}

The shape and size of the fuzzy subsets are different for each data set. They were chosen for technical reasons (to deal, for instance, with the inaccuracy of the sensors). This paper shows how fuzzy modeling was done for two different types of data: the yield and the soil depth. Fuzzy modeling of the other data is not detailed. However, sugar content, elevation, trunk diameters, and soil resistivity data were modeled similarly to yield data.

Previous work was done to manage inaccuracies of the DGPS [23] and the yield sensor [8]. We considered that a yield measurement might be taken into account within a radius of $1.5 \mathrm{~m}$ around the location given by the DGPS and should be taken into account within a $0.5 \mathrm{~m}$ area around the location. A yield value was defined as a fuzzy set with a support of $\pm 2 \%$ around the measured value and with a kernel of $\pm 1 \%$ around the measured value. Spatial and measurement accuracies were considered as shown in Fig. 3. For the other sensors, the expected inaccuracies were assessed from repetitions of measurements on several locations within the field.

The boundaries of the soil zones were hand drawn by the wine grower. The boundary of each zone was used to generate a fuzzy region. The support of the region was given by a dilation of the boundary and the kernel by an erosion. The distance between the kernel and the support was of $10 \mathrm{~m}$. The general shape of these fuzzy regions is described in Fig. 4a.

In this example, soil depth values are qualitative. Two labels were defined by the expert: shallow and deep. These two labels were associated with confidence degrees determined by the expert (4b), with respect to the usual property: $\Pi($ shallow $)+\Pi($ deep $) \geqslant 1$ (the expert could say that the soil was neither shallow nor deep).

Yield values were described using two qualitative levels (low and high). The other quantitative data were associated with three qualitative levels (low, medium and high). The breakpoints are percentiles of the given distributions (Figs. 5 and 6). Note that this method is based on the hypothesis that the number of low yield values is the same as the number of high yield values. Some other clustering methods (such as k-means) may be used. 
a

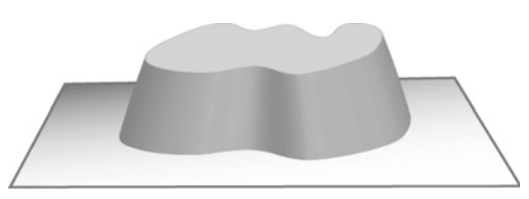

Location b

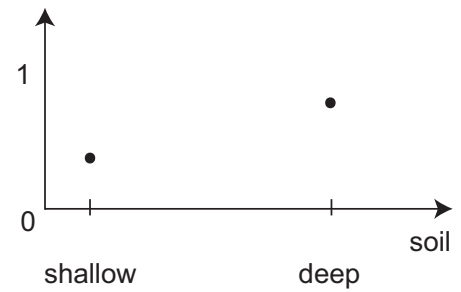

Qualitative label

Fig. 4. Description of a soil depth datum: (a) location; (b) qualitative label.

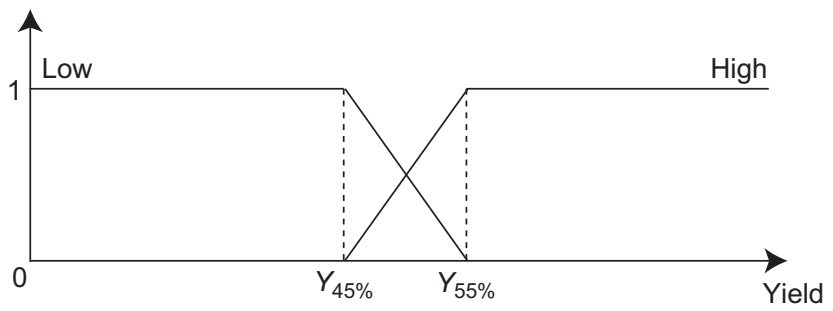

Fig. 5. Partition of the yield referential.

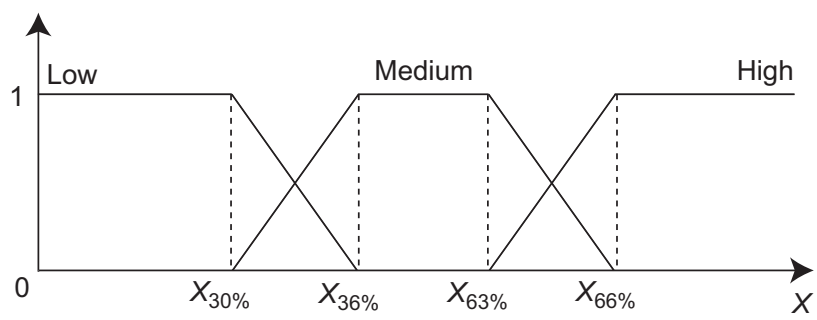

Fig. 6. Partition of a complementary measurement referential.

\subsection{Comparison with usual methods}

This section provides a comparison of the SBA method with two usual methods: kriging and fuzzy kriging. A yield data set was used to generate three yield maps (one per method). The comparison of the three maps was performed using specificity and similarity indexes.

\subsubsection{Method}

First, a regular grid of 1000 nodes $R_{i}$ was applied on the vineyard. Second, request zones were centered on each node of the grid. The definition of imprecise boundaries allowed us to consider the spatial continuity of within field variations. Their dimensions were defined according to the smallest area that could be considered, based on technical considerations (the size of the fertilizing machine, for instance). Each kernel contains four vine stocks. Each support contains all the neighboring kernels. The set of all the request zones defines a partition of the vineyard (Fig. 7).

Possibility degrees of low yield and high yield were computed on all the nodes of the grid using:

- kriging with local variograms,

- fuzzy kriging with a global variogram,

- semantic based aggregation method (SA method): in this case, the estimate of the node was provided by the estimate of the request zone centered on it. 


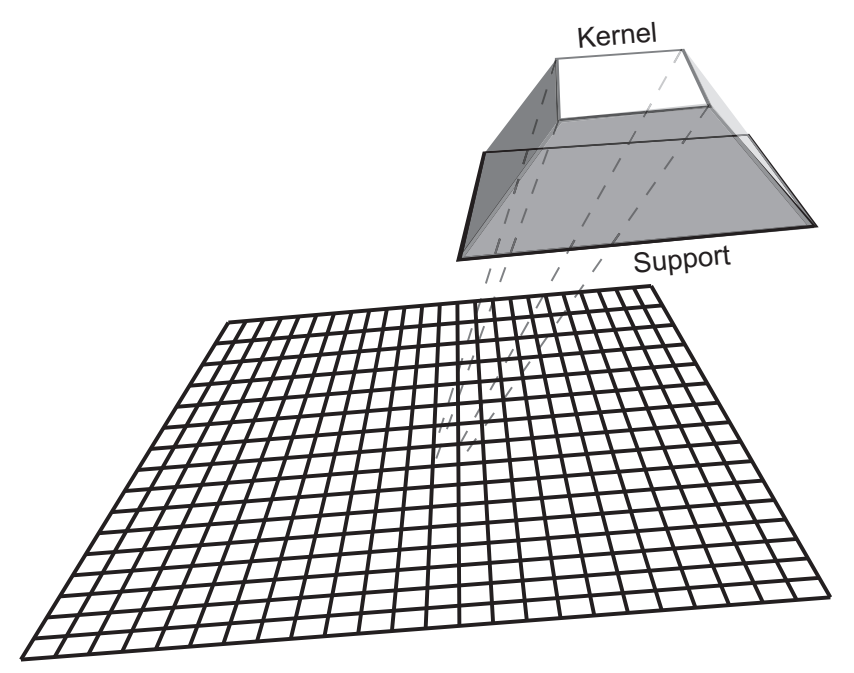

Fig. 7. Partition of the vineyard.

The kriging method is widely used to process spatial data [9]. It does not take into account any fuzzy subsets. It provides yield value estimates by linear combinations of measurements. The fuzzy kriging method aims at taking into account fuzzy measurements. It provides fuzzy yield values by linear combinations of fuzzy measurements (see Section 2 for details). For both methods, the computation of possibility degrees from yield values estimates or fuzzy yield values takes place after the estimation process. The SA method aims at taking into account both fuzzy measurements and fuzzy regions. It converts measured values into possibility degrees and then it aggregates them in order to directly provide estimates of possibility degrees for the request zone.

For the kriging method, estimation was performed using local variograms (Vesper software) [37]. For the fuzzy kriging method, the parameters of the global variogram were chosen manually, based on the experimental variogram. For the SA method, the parameters of the Choquet integral were set to an upper quartile with the lowest possible amount of filtering ( $\Delta=1$ and $\beta=0.75$ ). This couple of parameters was considered to be a reasonable choice, according to the empirical characteristics of the observations provided by the yield sensor. The sensitivity of the method to the parameters is studied in the next subsection.

For each method, we computed a specificity index (from 0 to 1 ) which indicates if the method associates uncertainty with their estimates or not. This index results from the arithmetic mean of Yager [48] specificity indexes computed on each node $\left(R_{i}\right)$ :

$$
\begin{aligned}
& S p\left(R_{i}\right)=\sum_{k=1}^{p} \frac{1}{k}\left(\Pi\left(A_{k} ; R_{i}\right)-\Pi\left(A_{k+1} ; R_{i}\right)\right), \\
& S p=\frac{1}{i} \sum_{i=1}^{m}\left(\operatorname{Sp}\left(R_{i}\right)\right), \\
& \Pi\left(A_{k+1}\right)=0, \\
& A_{1}=\text { low, } A_{2}=\text { high. }
\end{aligned}
$$

A similarity index which varies from 0 to 1 was defined in order to compare the different methods. This index allowed us to compare two methods at the time. This assessment was designed to let us know whether two methods provide the same results. As for the specificity indexes, the similarity indexes are arithmetic means of local values computed for 
a

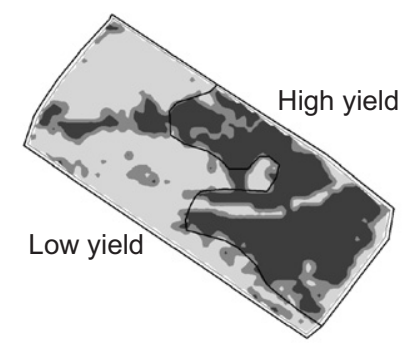

Kriging

C

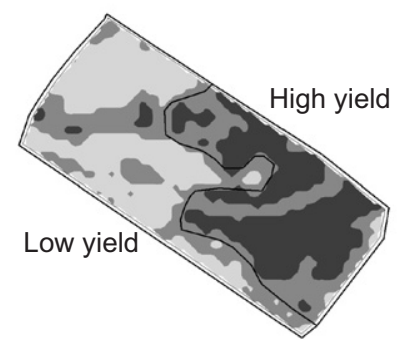

SA method b

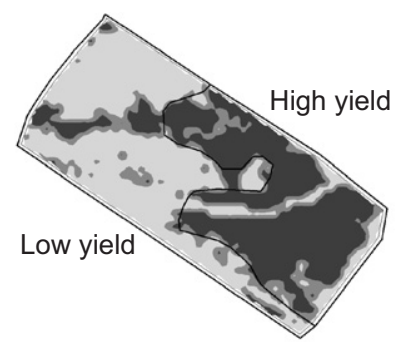

Fuzzy kriging

Fig. 8. Yield maps: (a) kriging; (b) fuzzy kriging; (c) SA method.

Table 1

Values of $S p$ for the different methods

\begin{tabular}{lll}
\hline Kriging & Fuzzy kriging & SA method \\
\hline 0.96 & 0.96 & 0.88 \\
\hline
\end{tabular}

each node $R_{i}$ :

$$
\begin{aligned}
& \operatorname{Sim}\left(R_{i}\right)=\max _{k \in[1, p]}\left(\min \left(\Pi_{\text {meth } 1}\left(A_{k} ; R_{i}\right) ; \Pi_{\text {meth } 2}\left(A_{k} ; R_{i}\right)\right)\right), \\
& \operatorname{Sim}=\frac{1}{i} \sum_{i=1}^{m}\left(\operatorname{Sim}\left(R_{i}\right)\right) \\
& A_{1}=\text { low }, \quad A_{2}=\text { high } .
\end{aligned}
$$

\subsubsection{Results and discussion}

Fig. 8 shows the three yield maps computed by the three different methods. Fig. 8a shows the map generated by the kriging method and Fig. $8 \mathrm{~b}$ shows the map generated by the fuzzy kriging method. The map resulting from the SA method is shown in Fig. 8c. These maps consider three types of management units:

- Dark zones, where the yield is high $(\mathrm{N}(h i g h ; R)=1)$.

- Gray zones, where the yield may be high or low $(\Pi(h i g h ; R)>0)$.

- White zones, where the yield is low $(\Pi(h i g h ; R)=0)$.

All the maps look similar. They highlight the same patterns of low and high yield. Table 1 provides the specificity indexes computed from the different methods. Table 2 shows the similarity indexes computed for all the couples of methods. 
Table 2

Values of Sim for the different method couples

\begin{tabular}{llll}
\hline & Kriging & Fuzzy kriging & SA method \\
\hline Kriging & 0.97 & 0.97 & 0.99 \\
Fuzzy kriging & 0.97 & 0.98 & 0.99 \\
SA method & 0.99 & 0.99 & 0.99 \\
\hline
\end{tabular}

Table 2 shows that all the methods provide similar estimates. Table 1 shows that all the methods provide high specificity indexes. However, the lowest specificity index is provided by the SA method. This specificity index must be related to the ability of the method to account for the imprecision and the uncertainty of the data. The results provided by the SA method are less specific than the results provided by kriging and fuzzy kriging because they account for the imprecision of measurements and locations.

This comparison with usual methods confirms our approach ability to account for both imprecisions and uncertainties. Note that unlike kriging and fuzzy kriging, our approach can consider qualitative information and can also be used when the number of data are low. Some of these aspects will be highlighted in the following subsections.

\subsection{Test of the ability of the SBA method to validate expert zones}

This subsection considers the ability of the SA method to validate an expert partition of the field. The yield data were aggregated in order to provide a qualitative estimate of each partition.

A sensitivity analysis was carried out for one request zone. It characterized the influence of the parameter on the result of the aggregation process.

\subsubsection{Method}

The expert proposed to simplify the partition of the field. In his opinion, he only needed to consider two homogeneous yield zones (instead of a thousand). From a technical point of view, this simplification of the partition provides a simplification of site-specific management. This subsection is dedicated to the delineation of the two yield zones and to the assessment of their relevancy.

The high yield zone and the low yield zone were drawn manually by the expert, see the limits in Fig. 10. We used the expert boundaries to generate fuzzy "request zones". As for the soil map, erosion and dilation were applied on the boundaries drawn by the expert in order to consider the spatial inaccuracy of the zone delineation. A distance of $20 \mathrm{~m}$ between the kernel and the support was considered in this study.

Both zones were considered as request zones in the next steps of the process. An aggregation of the yield information sources was performed on each zone in order to assess the relevancy of the expert delineation. The aggregation was performed with the same values as before $(\beta=0.75$ and $\Delta=1)$. A sensitivity analysis was conducted with different couples of parameters on the low yield zone.

\subsubsection{Results and discussion}

Table 3 shows the result of the aggregation of the yield information sources on the two expert zones. This table can be considered as a tool to assess the relevancy of the expert zoning. In our case, the "high yield" label is associated with a high necessity degree on the high yield zone, and the "low yield" label is associated with a high necessity degree on the low yield zone. Therefore, the expert's zoning is valid (with the acceptance of the $\beta$ and $\Delta$ parameters).

Fig. 9 shows the estimate of the possibility degrees of low and high yield on the low yield zone for different values of $\beta$ and $\Delta$. It highlights the influence of the parameters on the result of the aggregation process. The possibility degree of the "low" label equals 1 whatever the parameters. The possibility degree of the "high" label is a function of $\Delta$ and $\beta$. Low possibility degrees are provided with $\beta \in[0.55 ; 0.75]$ and $\Delta \in[1 ; 600]$. The variability of the result is more sensitive to $\beta$ modifications than to those of $\Delta$.

In possibility theory, a single piece of information can be used to compute a possibility degree. Therefore, it seems obvious that $\Delta$ has to be low (in this example, $\Delta \in[0 ; 50]$ is a reasonable choice). Fig. $9 \mathrm{~b}$ shows a breakpoint at around $\beta=0.75$. This result confirms the relevancy of the upper quartile in this case. Nevertheless, we have no reason to 
Table 3

Validation of the yield zones

\begin{tabular}{lllll}
\hline & $\Pi$ & & $\mathrm{N}$ & \\
\cline { 2 - 5 } \cline { 3 - 4 } & Low & High & Low & 1.000 \\
\hline Low yield zone & 1.000 & 0.000 & 0.000 & 0.000 \\
High yield zone & 0.000 & 1.000 & 1.000 \\
\hline
\end{tabular}

\section{a}

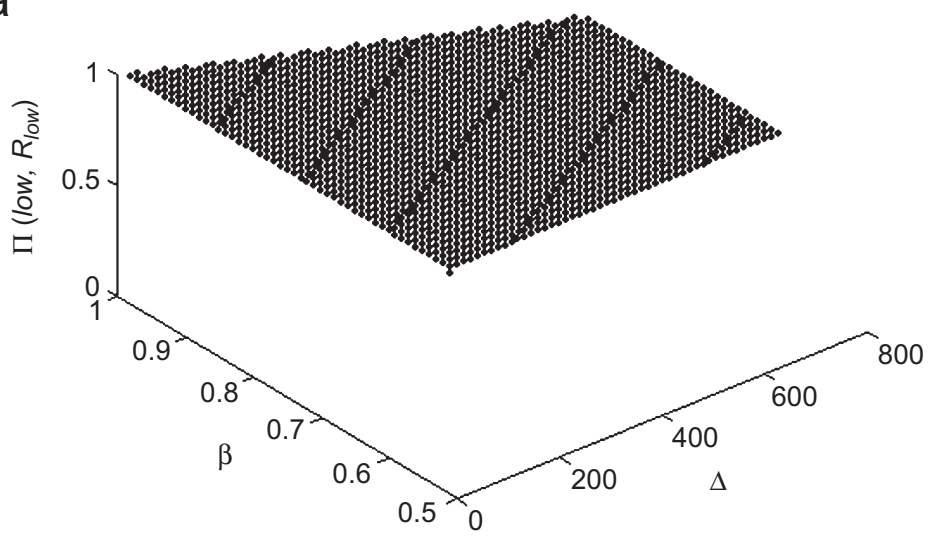

Possibility of low yield

\section{b}

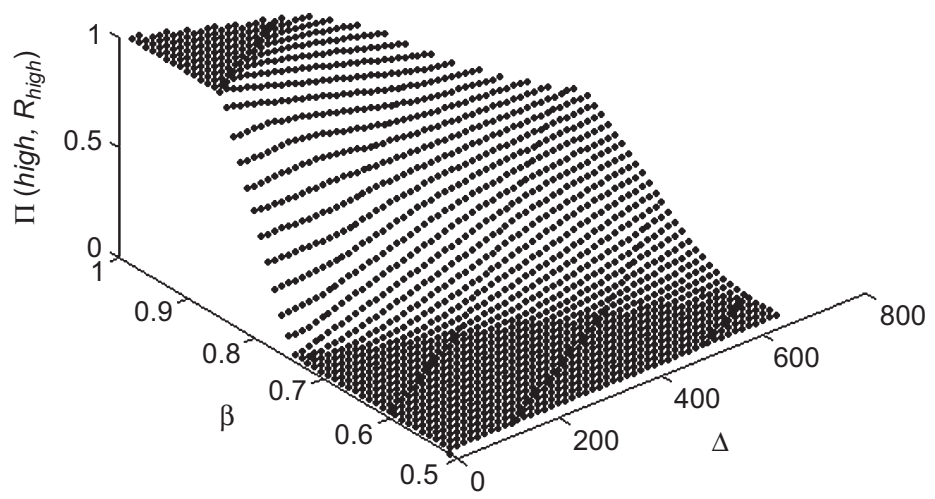

Possibility of high yield

Fig. 9. Estimate of possibility degrees for the low yield expert zone with different couples of parameters: (a) possibility of low yield; (b) possibility of high yield.

generalize the use of this value to other situations. The determination of $\beta$ is based mainly on an expert approach. However, the computation of cumulative histograms could be helpful.

\subsection{Qualitative estimate of the expert zones}

This subsection is dedicated to the qualitative estimate of each variable on each management zone. It shows the ability of our method to characterize expert zones using heterogeneous data sets. 

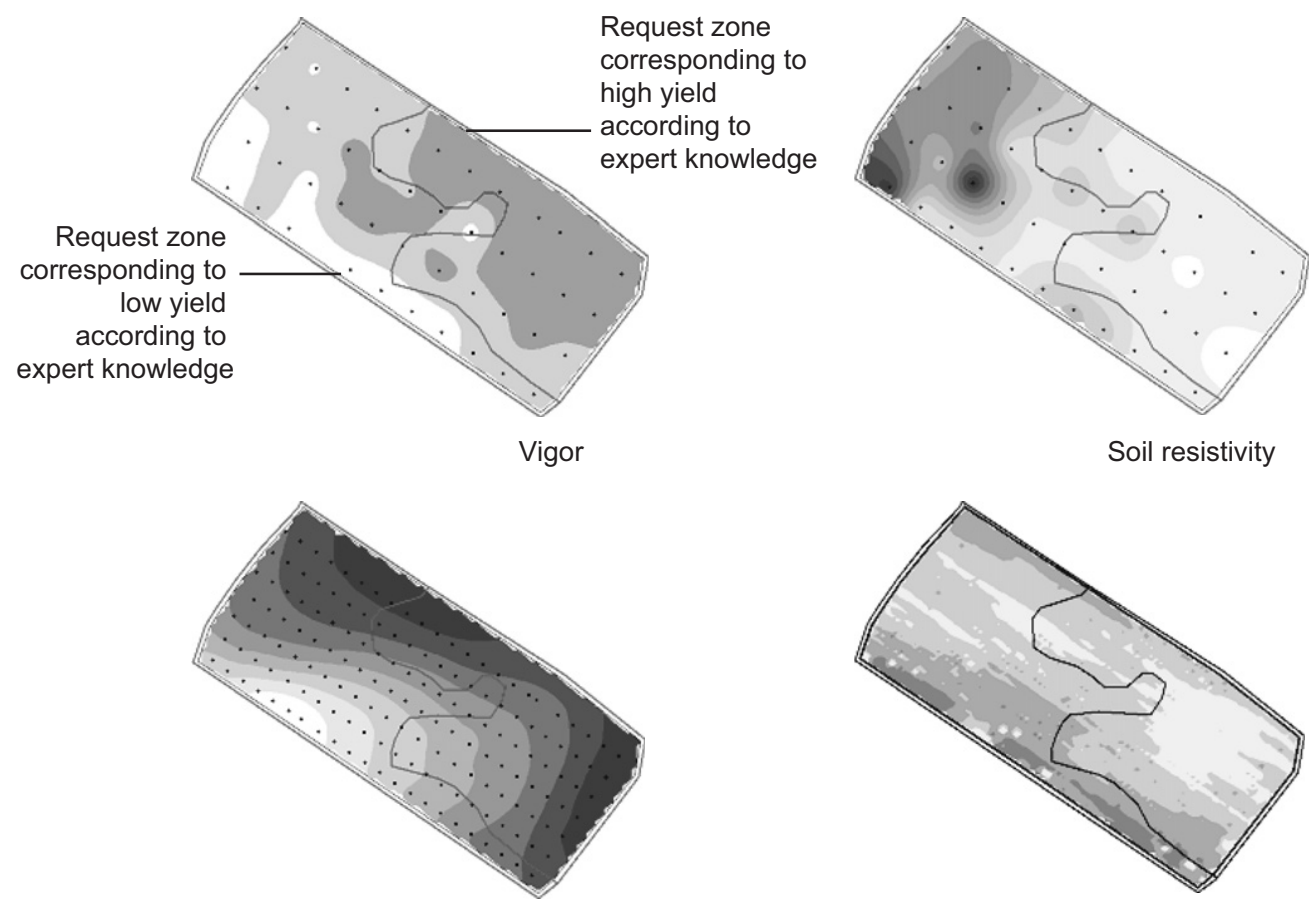

Elevation

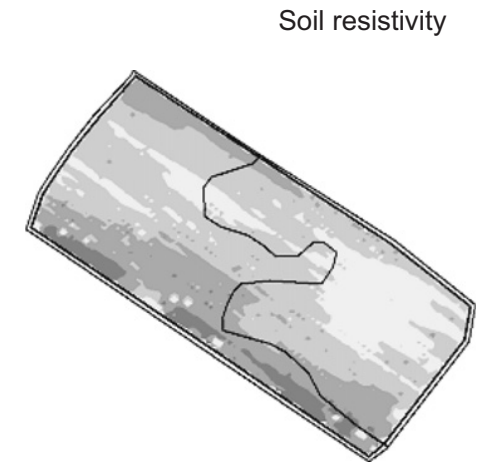

Sugar content

Fig. 10. Complementary measurements.

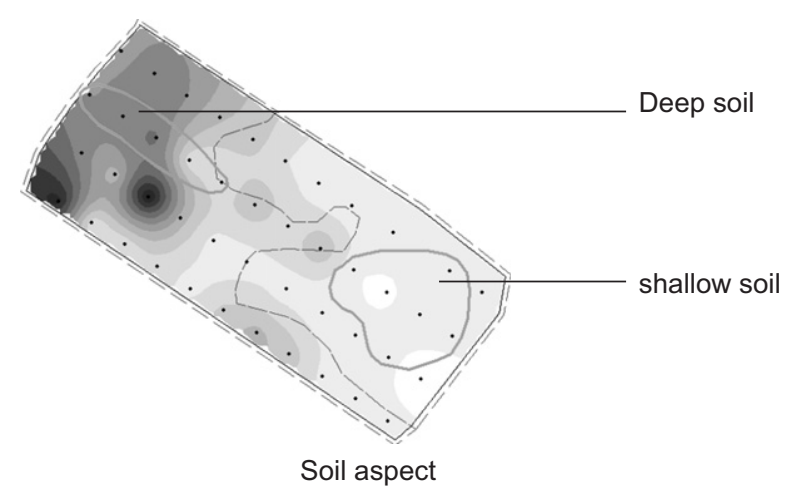

Fig. 11. Qualitative information about soil.

\subsubsection{Method}

Fig. 10 shows a basic representation of the spatial variations of the different variables. These maps were generated using a common kriging approach. For each map, the request zones delineated by the expert (high yield, low yield) were plotted. The sampling locations were also drawn. Fig. 11 shows the location of the soil expert zones. These zones were superimposed on the soil resistivity map.

The wine grower needs to understand what causes low yield in order to conduct site-specific management of the problem. However, to be able to make some assumptions on the vineyard, this wine grower needs to identify the parameters which can be considered as homogeneous on each yield zone, and the differences between the two yield zones. 
This identification is not straightforward and a simple observation of the different maps is not sufficient. To overcome this problem, a qualitative estimate of each parameter on each yield zone can be used. It may highlight trends for each zone and help the expert understand what is behind the low yield.

Once again, the aggregation was performed with $\beta=0.75$ and $\Delta=1$.

\subsubsection{Results and discussion}

On the low yield zone, the aggregation leads to:

- medium or high soil resistivity (but not low, $\Pi\left(\right.$ low $\left.; R_{\text {low }}\right)=0$ );

- medium or high sugar content (but not low, $\Pi\left(\right.$ low; $\left.R_{\text {low }}\right)=0$ );

- low or medium vigor (but not high, $\Pi\left(h i g h ; R_{\text {low }}\right)=0$ );

- low or medium elevation (but not high, $\Pi\left(h i g h ; R_{l o w}\right)=0$ );

- shallow soil (and possibly deep, $\Pi\left(\right.$ deep $\left.; R_{\text {low }}\right)=0.7$ ).

The result of the aggregation shows that low yield corresponds to particular conditions (soil, sugar content, elevation) that, taken together, confirm perennial soil water availability problems.

A similar process is performed on the high yield zone. The aggregation leads to:

- low or medium soil resistivity (but not high, $\Pi\left(h i g h ; R_{h i g h}\right)=0$ );

- low or medium sugar content (but not high, $\Pi\left(h i g h ; R_{h i g h}\right)=0$ );

- medium or high vigor (but not low, $\Pi\left(\right.$ low; $\left.R_{h i g h}\right)=0$ );

- medium or high elevation (but not low, $\Pi\left(\right.$ low; $\left.R_{\text {high }}\right)=0$ );

- deep soil (but not shallow, П(shallow; $\left.R_{\text {high }}\right)=0.2$ ).

Once again, high yield zones corresponds to specific conditions that, taken together, confirm that high amounts of available water is a perennial feature. The wine grower plans to manage the vineyard using an irrigation-based approach. The low yield zone will thus be irrigated.

This example shows how our method allows the aggregation of heterogeneous data on a zone of interest that makes sense for the expert. It shows how:

- Our approach may be used by a viticulturist to automatically partition a field into different management zones according to a given variable (i.e. the yield).

- Our method may also be used to ensure that zoning is pertinent according to available data.

- The zones made during the previous steps can be considered as spatial request zones to aggregate all the other variables.

- Our method gives a degree of certainty to the conclusion once the mono-variable aggregation process is performed.

- An estimate of several variables can be computed on a given zone, in order to give a multi-variable characterization of the request zone.

\section{Conclusion}

In this paper, we defined a new method for classifying request zones. This method (called semantic based aggregation method) considers pieces of information granulated on partitions which are then associated with semantic labels. It allows us to consider qualitative and quantitative data and to take into account both precision and confidence.

Each spatial datum is an information element, whose location and value are independently described. Locations are described by geographical fuzzy subsets, whereas values are described by qualitative labels defined using possibility and necessity degrees. A Choquet integral is used to aggregate the information elements, and to provide estimates of possibility and necessity degrees for any fuzzy request zone. The originality of our approach resides in the fact that it considers:

- the location of the information elements, with regard to the location of the request zone and of the other data,

- conflict between information elements by removing outliers from the aggregation process.

The SA method also accounts for the quantity of spatial information. It can be applied whatever the quantity of available data. However, the specificity of the estimate is dependent on the quantity of information at our proposal. When the 
number of available data is high, the results provided by the SA method are similar to those provided by kriging and fuzzy kriging. Its efficiency is illustrated on a precision viticulture data set for a field characterization. The example shows how the SA method has great potential to analyze spatial data in a way that is simple for an expert.

However, the estimation operator requires further work. More parameters have to be considered to describe the spatial distribution of the data, to account for the interactions between sets of information elements and request zones, and to determine the relevancy of a set of information elements in the description of a request zone.

\section{Acknowledgments}

This work was funded by the French ministry of research within the framework of the Eureka VITIS Project.

\section{References}

[1] M. Anile, B. Falciniedo, G. Gallom, M. Spagnuolo, S. Spinello, Modeling uncertain data with fuzzy b-splines, Fuzzy sets and systems 113 (2000) 397-410.

[2] M. Anile, P. Furno, G. Gallo, A. Massolo, A fuzzy approach to visibility maps creation over digital terrains, Fuzzy Sets and Systems 135 (1) (2003) 63-80.

[3] H. Auernhammer, Global positioning systems in agriculture, Comput. Electron. Agriculture 11 (1994) 1-95 (Special issue).

[4] H. Bandemer, A. Gebhardt, Bayesian fuzzy kriging, Fuzzy Sets and Systems 112 (3) (2000) 405-418.

[5] A. Bardossy, I. Bogardi, W.E. Kelly, Imprecise (fuzzy) information in geostatistics, Math. Geol. 20 (1988) $287-311$.

[6] A. Bardossy, I. Bogardi, W.E. Kelly, Kriging with imprecise (fuzzy) variograms i: theory, ii: application, Math. Geol. 22 (1990) 63-79, 81-94.

[7] S. Benferhat, D. Dubois, S. Kaci, H. Prade, Bipolar possibility theory in preference modeling: representation, fusion, and optimal solutions. Inform. Fusion 7 (1) (2006) 135-150.

[8] A. Bourely, Pellenc: la viticulture de précision, in: Actes des 11e journées du Groupe d'Etude des Systèmes de COnduite de la vigne (GESCO), Marsaala, Sicile, 1999, pp. 386-392.

[9] R. Bramley, A protocol for winegrape yield maps, in: S.G.G. Blackmore (Ed.), Proc. Third European Conf. on Precision Agriculture, Vol. 2, Agro Montpellier, 2001, pp. 173-767.

[10] R. Bramley, D. Lamb, Making sense of vineyard variability in Australia, in: Proc. Internat. Symp. on Precision Viticulture, Ninth Latin American Congr. on Viticulture and Oenology, 2003, pp. 35-54.

[11] P. Burrough, Natural objects with indeterminate boundaries, Geographic Objects with Indeterminate Boundaries, GISDATA, Vol. 2, Taylor Francis, London, 1996, pp. 3-28.

[12] D. Cazemier, P. Lagacherie, R. Martin-Clouaire, A possibility theory approach for estimating available water capacity from imprecise information contained in soil databases, Geoderma 103 (1-2) (2001) 113-132.

[13] G. Choquet, Theory of capacities, Ann. Inst. Fourier 5 (1953) 131-295.

[14] D.L. Corwin, S.R. Kaffka, J.W. Hopmans, Y. Mori, J.W. van Groenigen, C. van Kessel, S.M. Lesch, J.D. Oster, Assessment and field-scale mapping of soil quality properties of a saline-sodic soil, Geoderma 114 (3-4) (2003) 231-259.

[15] P. Diamond, Fuzzy kriging, Fuzzy Sets and Systems 33 (3) (1989) 315-332.

[16] T. Doerge, Defining management zones for precision farming, Crop Insights 8 (1998).

[17] S. Dragićević, Multi-dimensional interpolations with fuzzy sets, in: F. Petry, V. Robinson, M. Cobb (Eds.), Fuzzy Modeling with Spatial Information for Geographic Problems, Springer, Berlin, 2005, pp. 143-158.

[18] S. Dragićević, D. Marceau, An application of fuzzy logic reasoning for GIS temporal modeling of dynamic processes, Fuzzy Sets and Systems 113 (1) (2000) 69-80.

[19] D. Dubois, Possibility theory and statistical reasoning, Comput. Statist. \& Data Anal. 51 (1) (2006) 47-69.

[20] D. Dubois, H. Prade, Possibility Theory, Plenum Press, New York, 1988.

[21] D. Dubois, H. Prade, On fuzzy interpolation, in: Proc. Third Conf. on Fuzzy Logic, Neural Networks and Soft Computing, Japan, 1994.

[22] T. Ebertseder, R. Gutser, U. Hege, R. Brandhuber, U. Schmidhalter, Strategies for site-specific nitrogen fertilization with respect to long-term environmental demands, in: J. Stafford, A. Werner (Eds.), Proc. Fourth European Conf. on Precision Agriculture, Berlin, 2003 , pp. $193-198$.

[23] M. Ehrl, W. Stempfhuber, H. Auernhammer, M. Demmel, Quality assessment of agricultural positioning and communication system, in: J. Stafford, A. Werner (Eds.), Proc. Fourth European Conf. on Precision Agriculture, Vol. 1, Wageningen Academic, 2003 , pp. $205-210$.

[24] M. Grabisch, T. Murofushi, M. Sugeno, Fuzzy Measures and Integrals-Theory and Applications, Studies in Fuzziness and Soft Computing, Physica-Verlag, Heidelberg, New York, 2000.

[25] G. Grenzdörffer, Requirements and possibilities of remote sensing for precision agriculture—current status and future developments, in: S. Blackmore, G. Grenier (Eds.), Proc. Third European Conf. on Precision Agriculture, Vol. 1, 2001, pp. 211-216.

[26] G. Grenzdörffer, Investigations on the use of airborne remote sensing for variable rate treatments of fungicides, growth regulators and n-fertilisation, in: J. Stafford, A. Werner (Eds.), Proc. Fourth European Conf. on Precision Agriculture, Berlin, 2003, pp. 241-246.

[27] P. Huber, Robust statistics, Wiley, New York, 1981.

[28] A. Journel, C. Huijbregts, Mining Gesotatistics, Academic Press, London, 1978.

[29] R. Kerry, M. Oliver, Comparing spatial structures in soil properties and ancillary data by using variograms, in: S. Blackmore, G. Grenier (Eds.), Proc. Third European Conf. on Precision Agriculture, Vol. 1, Agro Montpellier, 2001, pp. 413-418. 
[30] R. Kerry, M. Oliver, Maximum likelihood variograms for efficient prediction in precision agriculture, in: J. Stafford (Ed.), Proc. Fifth European Conf. on Precision Agriculture, Wageningen Academics Publishers, Uppsala, Sweden, 2005, pp. 707-714.

[31] C. Kvien, S. Pocknee, Introduction to why management zones, Technical Report, University of Georgia's College of Agricultural and Environmental Sciences, 2000.

[32] P. Lagacherie, D. Cazemier, R. Martin-Clouaire, T. Wassenaar, A spatial approach using imprecise soil data for modelling crop yields over vast areas, Agriculture Ecosystems Environm. 81 (2000).

[33] W. Lodwick, J. Santos, Constructing consistent fuzzy surfaces from fuzzy data, Fuzzy Sets and Systems 135 (2) (2003) $259-277$.

[34] Marichal, J.-L., Aggregation operators for multicriteria decision aid, Ph.D. Thesis, Université de Liège, Faculté des sciences, 1999.

[35] R. Martin-Clouaire, D. Cazemier, P. Lagacherie, Representing and processing uncertain soil information for mapping soil hydrological properties, Comput. Electron. Agriculture 29 (1-2) (2000) 41-57.

[36] G. Matheron, The theory of regionalized variables and its applications, Les cahiers du Centre de Morphologie. Centre de Géostatistiques de Fontainebleau, Vol. 5, 1970.

[37] B. Minasny, A. McBratney, B. Whelan, Vesper version 1.6. (Variogram Estimation and Spatial Prediction plus ERror)—Australian Centre of Precision Agriculture, The University of Sydney 〈http://www.usyd.edu.au/su/agric/acpa/vesper/vesper.html〉, 2002.

[38] M. Molenaar, T. Cheng, Fuzzy spatial objects and their dynamics, Photogrammetry Remote Sensing 55 (2000) 64-175.

[39] T. Murofushi, M. Sugeno, An interpretation of fuzzy measure and the Choquet integral, Fuzzy Sets and Systems 29 (1989) $201-227$.

[40] T. Murofushi, M. Sugeno, Some quantities represented by the Choquet integral, Fuzzy Sets and Systems 56 (1993) $229-235$.

[41] R. Ortega, A. Esser, O. Santibaez, Spatial variability of wine grape yield and quality in chilean vineyards: economic and environmental impacts, in: J. Stafford, A. Werner (Eds.), Proc. Fourth European Conf. on Precision Agriculture, Berlin, Germany, 2003, pp. 499-506.

[42] J.A. Piotrowski, F. Bartels, A. Skalski, G. Schmidt, Geographical regionalization of glacial aquitard thickness in northwestern Germany, based on fuzzy kriging, Math. Geol. 28 (1996) 437-452.

[43] M. Schneider, Uncertainty management for spatial data in databases: fuzzy spatial data types, in: Sixth Internat. Symp. on Advances in Spatial Databases (SSD), Springer, Berlin, 1999, pp. 330-351.

[44] M. Sugeno, Theory of fuzzy integrals and its applications, Ph.D. Thesis, Tokyo Institute of Technology, 1974.

[45] J. Taylor, Digital terroirs and precision viticulture: investigations into the application of information technologies in Australian vineyards, Ph.D. Thesis, University of Sydney, 2004.

[46] J. Taylor, B. Tisseyre, R. Bramley, A. Resid, A comparison of the spatial variability of vineyard yield in European and Australian production systems, in: J. Stafford (Ed.), Proc. Fifth European Conf. on Precision Agriculture, Wageningen Academic Publishers, Uppsala, Sweden, 2005.

[47] B. Tisseyre, C. Mazzoni, N. Ardoin, C. Clipet, Yield and harvest quality measurement in precision viticulture-application for a selective vintage, in: S. Blackmore, G. Grenier (Eds.), Proc. Third European Conf. on Precision Agriculture, Vol. 1, Agro Montpellier, 2001, pp. 133-138.

[48] R.R. Yager, Measuring tranquility and anxiety in decision making: an application of fuzzy sets, Internat. J. Man-Machine Stud. 8 (3) (1982) 139-146.

[49] L. Zadeh, Fuzzy sets, Inform. and Control 8 (1965) 338-353.

[50] L. Zadeh, Fuzzy sets as a basis for a theory of possibility, Fuzzy Sets and Systems 1 (1978) 3-28.

[51] L. Zadeh, Toward a theory of fuzzy information granulation and its centrality in human reasoning and fuzzy logic, Fuzzy Sets and Systems 90 (1997) 111-127.

[52] A. Zhu, Measuring uncertainty in class assignment for natural resource maps under fuzzy logic, Photogrammetric Engng. Remote Sensing 63 (1997) 1195-1202.

[53] A. Zhu, A similarity model for representing soil spatial information, Geoderma 77 (1997) 217-242.

[54] A. Zhu, B. Hudson, J. Burt, K. Lubich, D. Simonson, Soil mapping using GIS, expert knowledge, and fuzzy logic, Soil Sci. Soc. Amer. J. 65 (2001) 1463-1472. 